\title{
Integral geometry methods on deformed categories in field theory II
}

\author{
Francisco Bulnes \\ Head of Research Department, Research Department in Mathematics and Engineering, TESCHA, Federal Highway, Mexico-Cuautla Tlapala \\ "La Candelaria", Chalco, P. C. 56641, Mexico
}

\section{Email address:}

francisco.bulnes@tescha.edu.mx

\section{To cite this article:}

Francisco Bulnes. Integral Geometry Methods on Deformed Categories in Field Theory II. Pure and Applied Mathematics Journal. Special Issue: Integral Geometry Methods on Derived Categories in the Geometrical Langlands Program. Vol. 3, No. 6-1, 2014, pp. 1-5. doi: $10.11648 /$ j.pamj.s.2014030602.11

\begin{abstract}
The integral geometry methods are applied on deformed categories to obtain correspondences in the geometrical Langlands program and construct the due equivalences between geometrical objects of the moduli stacks and algebraic objects of the corresponding categories and their $\mathrm{L}_{\mathrm{G} \text {-opers }}$ characterizing the solution classes to field theory equations in the belonging cohomological context such as $\left.H^{0}(\mathrm{~g}[\mathrm{z}]], \mathrm{V}_{\text {critical }}\right)=\mathrm{C}\left[0 \mathrm{p}_{L G}\left(\mathrm{D}^{\mathrm{X}}\right)\right]$ which is natural in the framework of the integral transforms to the generalizing of the Zuckerman functors that will be useful to the obtaining of the different factors of the universal functor of derived sheaves of Harish-Chandra to the Langlands geometrical program in mirror symmetry. The cosmological problem that exists is to reduce the number of field equations that are resoluble under the same gauge field (Verma modules) and to extend the gauge solutions to other fields using the topological groups symmetries that define their interactions. This extension can be given by a global Langlands correspondence between the Hecke sheaves category $\mathrm{H}_{\mathrm{G}^{\wedge}} \infty$ on an adequate moduli stack and the holomorphic $L_{\mathrm{G}-}$ bundles category with a special connection (Deligne connection). The corresponding $D_{-}$modules may be viewed as sheaves of conformal blocks (or co-invariants) (images under a generalized version of the Penrose transform) naturally arising in the framework of conformal field theory.
\end{abstract}

Keywords: Langlands Correspondence, Hecke Sheaves Category, Moduli Stacks, Ramifications, Twisted $D$ _ Modules, Verma Module Extensions

\section{Introduction}

The study of integral geometry methods have can establish different aspects on invariant theory and cohomology of cycles to study of the Universe or their parts. Spaces of equivalence modulo a relation of congruence are constructed on field solutions to establish a theory of the universe that includes the theory QFT (Quantum Field theory), the SUSY (Super-symmetry theory) and HST (heterotic string theory) using the sheaves correspondence of differential operators of the field equations and sheaves of coherent $D$-Modules [1]. The above mentioned correspondence use a Zuckerman functor that is a factor of the universal functor of derived sheaves of Harish-Chandra to the Langlands geometrical program in mirror symmetry [2, 3, 4]. The obtained development includes complexes of $D$-modules of infinite dimension, generalizing for this way, the BRST - cohomology in this context. With it, the class of the integrable systems is extended in mathematical physics and the possibility of obtaining a general theory of integral transforms for the space - time (integral operator cohomology [5]), and with it the measurement of many of their observables [6]. Also tends a bridge to complete a classification of the differential operators for the different field equations using on the base of Verma modules that are classification spaces of $S O(1, n+1)$, where elements of the Lie algebra $\mathrm{sl}(1, n+1)$, are differential operators, of the equations in mathematical physics [1].

\section{Deformed Categories and Penrose Transforms Correspondences on Ramifications}

First, the integral transforms when are used to establish isomorphisms between moduli spaces involve Lagrangians that come from the actions of the field in the quantized twistor space [1] and that have the order of have included to the HST (Heterotic String Theory), inside a twistor-string theory $[1,3]$ 
that could realize the bridge between the BRST - cohomology and the Lie algebra cohomology with results consigned from the quantum resolutions of the QFT, but considering the hypothesis given in the Kashiwara's conjectures to satisfy in this respect to the holonomicity and conformally properties (we want to discompose singular regions of the space-time in regions of conformal nature that can give the values of uniformity calculated as zeros of polynomials (Artin rings) in mathematical physics and re-interpreted by the Penrose transform as varieties (algebraic manifolds) in algebraic geometry) that involve phenomena of irregular singularities as fermion phenomena, particles decaying using rational curves. One way have been obtained by the use of the Penrose-Ward transform in the sense of Hecke categories where the $\widetilde{g}-$ modules shape an affine Kac-Moody algebra which is the universal one-dimensional central extension of the loop algebra $g(t))$, this last, deduced from the orbits that appear in the category $\mathrm{D}(L G)$, which is the smooth group algebra [2]. Why is important this identifying? In the measure that we be able establish to all the levels of the space-time the closure of the grupoid operation in every element of the $G$-invariant coherent $D$-modules category, we can tack the space-time through of the different moduli stacks and obtain the different carpets of the space-time corresponding to every one of the field interactions, that shape the quantum carpet (method of the corollary in [3]). One identification obtained by this method is the found in the paper given in [1], where using some integral operator cohomologies, through of one of their invariant of field is obtained after, a fundamental electromagnetic carpet of the space-time of a photon framework.

If we consider $\mathrm{H}_{G}=\mathrm{M}\left(\mathrm{D}_{\lambda}^{G / H}\right), \forall \lambda \in \mathrm{h}^{*}$, (as was done in [2, 3]) then $\mathrm{H}_{G}=\mathrm{M}(\mathrm{B} \backslash G / \mathrm{B})$, from the group $G=G^{\mathrm{C}}$. B - equivariant $D$-module on the flag manifold $X=G / \mathrm{B}$, provide integral kernels and thus integral transforms, to know

$$
H^{0}\left(X, L_{\lambda}\right) \cong \operatorname{ker}\left(\tilde{U}, Q_{\mathrm{BRST}}\right)
$$

where $Q_{\mathrm{BRST}}=\delta+\Phi_{1}^{i} \theta_{1}-\Phi_{2}^{i} \theta_{1}$, such that $Q_{\mathrm{BRST}}^{2} \phi=0$, where $\Phi_{1}^{i}$ and $\Phi_{2}^{i}$, are Higgs fields on either side of the open string [4].

Our demonstration was realized using the fact that each one of these subspaces of the complexes Cousin must be Hecke eigen-sheaves. The sum of Hecke eigen-sheaves is the Hecke category in our result, which is twisted, since there are images of isomorphisms of Harish-Chandra modules (that is to say, in a category of Harish-Chandra whose $D$-modules are $D_{\mathrm{F}}-$ modules) created via the Penrose transform on twisted modules. Here the property of being twisted is demonstrated by the argument of the Penrose transform that involve to the twistor transform in field theory. The twistor transform followed of Penrose $P(T)$, evaluates the kernels of the cohomological groups that are isomorphisms in the sense of the equivalences of the Kashiwara theorem [3].

Precisely the equivalences given in (1) shape a classification given of the homogeneous vector bundles of lines $[2,5]$ to a certain sector of differential operators obtaining an classification by Verma modules that haves that to see with the Recillas conjecture [1,2] and the Szegö kernels associated with Harish-Chandra modules [1]. But is necessary to realize some extensions in the same context of the $D_{\mathrm{P}}-$ modules transform conserving the confomally in certain level, through the language of the stacks, that is to say, we want extend our research al context of non-conformal manifolds and with it to find some condition at least of sufficiency for the resolution via one generalized Penrose transform of $D_{\mathrm{M}}-$ Modules, which are $D_{\mathrm{P}}-$ Modules and to induce their application to para-conformal classes through of the use of versions more generalized of Radon transforms that are connected with Penrose transforms to create conformal invariance in geometrical images that are physical stacks at least in the first development and tries to obtain an integral operators cohomology of the type $H^{\bullet}(X, \mathrm{O})$ [5] (X a complex variety with regular singularities) that be a sheaf cohomology group that includes the BRST - cohomology studied in field theory and where be applicable the second scheme of gauge theories (topological gauge theory $[4,7]$ ).

Then the equivalences of (1) in the $D_{G / H}-$ modules categories and to the functors $\Phi_{\mathrm{K}}^{0} \mathrm{~F}, \mathrm{G} \Psi_{\mathrm{K}}^{0}, \forall \mathrm{K}$, a $D_{\mathrm{P}}$ - module that is a $D_{G / H}$ - module, said isomorphism are translated in

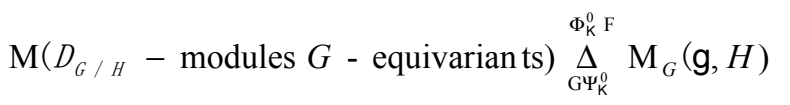

which must be translated in the isomorphism the Hecke categories

$$
\mathrm{H}_{G^{\wedge}} \Delta \mathrm{M}_{\mathrm{K}}(\tilde{\mathrm{g}}, \mathrm{Y})
$$

under duality of the cycles on $G(t)$ (B-equivariant $D-$ modules on the flag variety $G / \mathrm{B}$ ) and using their images of kernels corresponding to the integral transforms. The Lie algebra $\widetilde{\mathrm{g}}$, is the loop extension of the loop algebra $\mathrm{g}(t)$.

Here $\mathrm{M}\left(D_{G / H}-\right.$ modules $G$-equivariants $)$, is the full subcategory of $\operatorname{Mod}_{\text {coh }}\left(D_{X}\right)$, whose open sets are sets of the sheaf type $\mathrm{D}\left(\operatorname{Bun}_{G}(\Sigma)\right)$, to some bundle of lines $\operatorname{Bun}_{G}(\Sigma)$. The manifold ${ }_{\mathrm{Y}}$, is a set of isomorphic orbifolds to the corresponding loop quotient sub-classes of the homogeneous space $G / K$ (Applying the localization functors to these categories, and their $K$ - equivariant subcategories $\mathrm{M}\left(\widetilde{\mathrm{g}}_{\mathrm{k}_{\mathrm{c}}}, \chi\right)$, for various subgroups $K \subset G[[t]]$, we obtain categories of Hecke eigensheaves on the moduli spaces of $G$-bundles on $X$, with critical level (or parabolic structures) where $K$, is a "compact" subgroup of $G((t))$, which is the complex loop group corresponding to the loop algebra $\mathrm{g}(t)$.

Remember that the geometrical hypothesis in the functor $\Phi+$ Geometrical hypothesis, comes established for the geometrical duality of Langlands which says that the derived

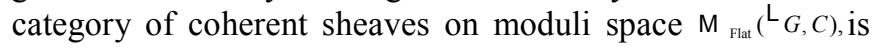
equivalent to the derived category of D-modules on the moduli space Bun $_{G}(C)$. Now our moduli space will be $\mathrm{D}\left(\mathrm{Bun}_{G}(C)\right)$. If we want generalize the results foreseen in this context of the derived categories to the ramifications of Langlands, the idea is to obtain equivalences between the different $D_{G / H}-$ modules classes establishing the isomorphisms between categories whose Zuckerman functor 
let be restricted to the sub-category of left $G$-equivariant $D_{G / H}$ - modules that are product of a trianguled sub-category as the given in $[1,6]$, and for a factor category that comes from certain Zuckerman functor constructed from image of one derived category using the corresponding generalization of the integral transforms on $D$-modules $[8,9,10,11]$.

Of this way, and using extensions of Verma modules is obtained the result:

Theorem 2.1 (F. Bulnes). The derived category of quasi$G$-equivariant $-D_{G / H}$ - modules shaped with the generalized and extended Verma modules given by ${ }^{L} \Phi^{\mu}(\mathrm{M})=\mathrm{M} \mathrm{T} \rho^{\mu}(\mathrm{V})$, $\forall \mathrm{V} \in\left(\operatorname{Loc}_{L}\right)$, can be identify for critically twisted sheaf category of $D$-modules on moduli stack Bun $_{G, y}, \forall y \in X$ (singularity) identified by the Hecke category $\mathrm{H}_{G, \mathrm{~K}, y}$ (geometric Langlands correspondence) if this is an image of integral transforms acting on ramifications of the Hecke category $\mathrm{H}_{G}, \forall \lambda \in \mathrm{h} *$ (for example $\mathrm{H}_{G, \lambda}$ ) on the flag variety $G / \mathrm{B}$, with weight corresponding to twisted differential operators on $\mathrm{Bun}_{G, y}$.

Proof. [2].

Table 1. Some Hecke categories and their ramifications.

\begin{tabular}{|c|c|c|}
\hline Derived Twisted Category $\mathrm{H}_{G, \lambda}$ & Geometrical Image: Holomorphic ${ }^{L} G-$ bundles & Co-cycles \\
\hline $\mathrm{H}_{G, \lambda}^{a f f}$ & $\mathrm{C}^{\times}-$Bundle & $H \backslash \widetilde{G} / K$ Complex Hyper-lines \\
\hline $\mathrm{H}_{G} \cong(B \backslash G / B)$ & $D^{\times}-$Bundle & $B \backslash \widetilde{G} / B$ Deformable Cycles or Orbifolds \\
\hline$H_{G}^{m o n} \cong(N \backslash G / N), \lambda=0$ & $\tilde{D}^{\times}-$Bundle & $N \backslash \widetilde{G} / N$ Hyper-planes or hypersurfaces \\
\hline $\mathrm{H} \cong\left(G(F) \backslash G\left(\mathrm{~A}_{F}\right) / G(\mathrm{O})\right)$ & $\mathrm{D}_{\mathrm{BRST}}-$ Bundle & $G(F) \backslash G\left(\mathrm{~A}_{F}\right) / G(\mathrm{O})$ Strings \\
\hline
\end{tabular}

The application of the Penrose transform on ${ }^{L}$ Bun $_{G}$, let see which are generalized Verma modules of the Langlands geometrical equivalences $\mathrm{D}_{\text {BRST }}\left(\operatorname{Oper}_{L_{G}}^{\leq n}\left(\mathrm{D}_{y}\right)\right) \cong \mathrm{D}^{\times}\left(\operatorname{Bun}_{G}(\Sigma)\right)$, which are demonstrated in [2]. But by the conjecture 8 , given in [12], the unique objects that are coefficients of the cohomological space of dimension $0,\left(H^{0}(X, O)\right)$, are the Verma modules $\mathrm{V}_{\lambda}(\chi), \forall \chi \in \mathrm{Op}_{{ }_{L_{G}}}{ }^{\lambda}$.

But $\mathrm{D}^{\mathrm{b}}\left(\widetilde{\mathrm{g}}_{\mathrm{K}_{c}}-\bmod _{\chi}\right) \cong \mathrm{D}^{\mathrm{b}}\left(Q \operatorname{Coh}\left(\operatorname{Sp}_{u}^{\mathrm{D} G}\right)\right), 1$ then the Hecke category is of the Verma modules ${ }^{L} \Phi^{\mu}\left(\bigvee_{w(\lambda+\rho)-\rho}\left(\chi_{y}\right)\right), \forall w \in W$, which are the ramifications of the Hecke category $\mathrm{H}_{G}, \forall \lambda \in \mathrm{h} *$.

To know explicitly the cycles and cocycles, and under geometrical arguments of their Penrose transforms we can consult the Table 1.

After we generalize the functors $R \Gamma_{K}^{H}$, to the functors ${ }^{L} \Phi_{\mathrm{T}}^{\mu}$, we obtain certain correspondences between cycles and co-cycles on derived categories of corresponding $D_{\mathrm{P}}-$ modules that are quasi-coherent $D_{\lambda}$ - modules (with the corresponding character of Hecke category $[12,13,14,15])$ whose deformed image correspond to some ramifications [16] which can be classified by the following theorem given in [17]:

Theorem 2.2 (F. Bulnes). Consider the classes of Hecke category $\mathrm{H}_{G^{\wedge}, \infty}, 2$ satisfying the geometrical Langlands correspondences given by

$$
\mathrm{D}_{\text {BRST }}\left(\operatorname{Oper}_{L_{G}}^{\leq n}\left(\mathrm{D}_{y}\right)\right) \cong \mathrm{D}^{\times}\left(\operatorname{Bun}_{G}(\Sigma)\right)
$$

(as was established in [2]). The unique geometrical pictures in field theory to different cohomological classes of the sheaves in $\mathrm{D}^{\times}\left(\operatorname{Bun}_{G}(\Sigma)\right)$, are the co-cycles (images under

1 Derived category of quasicoherent sheaves on a suitable $D G$.

2 Here $\mathrm{H}_{G^{\wedge}, \infty}$, is the Hecke category that assigns the $\infty-$ categories of quasi-coherent sheaves on the stack $\operatorname{Loc}_{{ }^{L} G}$, of $\Sigma$. Also this Hecke category is $\mathrm{H}_{G, \lambda} \forall \lambda \in \mathrm{h}^{*}$, that is the twisted Hecke category consisting of the natural integral transforms acting on categories of $\lambda$-twisted $D$-modules of the flag variety $G /$ B (see the theorem 2. 2). This theorem also comes to strengthen the conclusion obtained in the theorem 3. 1, and establish a correspondence between the open curves of the moduli stacks of $G$-bundles as Bun $_{G, y}$, and Hecke algebras. $G(t)$, is the loop group that acts naturally on every one the categories $\mathrm{M}_{\mathrm{K}_{c}}(\widetilde{\mathrm{g}}, \mathrm{Y})=\widetilde{\mathrm{g}}_{\mathrm{K}_{c}}-\bmod _{\chi}$, in the Frenkel notation $[12,15,16]$. different Penrose transform versions):

a) Orbi-folds or,

b) Strings as twisted hyper-lines and twisted hyper-planes or,

c) Super-twistor surfaces (from complex 2- and 3-dimension spaces). Proof. [17].

Then due the generalized geometries that can be obtained through the $D_{G / H}$ - modules (for the mirror theory induced by the Hitchin moduli space $[4,19])$ and considering the Higgs nature of the fields obtained by this procedure [1, 17], was establish the following result:

Theorem (F. Bulnes) 2. 3. Considering $\mathrm{M}_{\text {Higgs }}(G, C)=T_{V}^{\vee} \operatorname{Bun}_{C}(\Sigma)$, and $\left.\phi\right|_{\left.C x_{i} \theta\right\}}=\theta$, defined before we have

$$
\mathrm{M}\left({ }^{L} G, C\right)=\mathrm{M}_{\text {Higgs }}\left({ }^{L} G, C\right) K^{1 / 2},
$$
wher e $K^{1 / 2}$, is the square root of the bundle of lines on $\mathrm{Bun}_{G}$, corresponding to the critical level. Proof. [2, 3].

What happen with the ramifications $\nabla_{s}$, to the case $H^{*}\left({ }^{L} G, \tilde{\mathrm{L}_{\lambda}} \otimes P^{*}\left(K^{1 / 2}\right) \oplus \mathrm{L}^{\otimes 2}\right)$ ? Geometrical correspondences cannot be determined because $\nabla_{s}$, is not holomorphic?

We can consider some extensions of Moduli stacks and a corresponding induced bundle of lines.

From the Theorem 4. 1, is clear that the ramification to the part of connection $\nabla_{s}$, must be inside the context of the moduli space $\left.\mathrm{M}_{\text {Higgs }}{ }^{L} G, C\right)$. The induced lines bundle must be one from $T_{V}^{\vee} \operatorname{Bun}_{C}(\Sigma)$, with the condition of that it must be a divisor of holomorphic vector bundle.

At the respect, is had the following theorem [2] in which is considered the results demonstrated in [18, 19], and some ideas of the seminar work given in [15]:

Theorem (F. Bulnes). 2. 4. If $\nabla_{s}$, has moduli stack $\tilde{L}_{\lambda}=L^{\otimes 2}$, where $L^{\otimes 2}$, is the sub-bundle of lines

$$
\mathrm{L}^{\otimes 2} \cong \tilde{\mathrm{L}}_{\left[\bar{C}_{h_{V}}(\theta)\right]} \otimes \zeta^{\otimes-(n-1)}
$$

where $\bar{C}_{V} \rightarrow C \times T_{V}^{\vee}$ Bun, is simply the cover of $\left(p_{C}^{*} V, \phi\right)$, and hence comes equipped with a natural line bundle ${\tilde{L_{\lambda}}}_{\lambda}$, such that $\pi_{V^{*}} \tilde{L}_{\lambda}=p_{C}^{*} V$, then their generalized Penrose transform (which is a 
Penrose-Ward transform) comes given by

$$
H^{0}\left({ }^{L} G, \Gamma(U, \mathrm{O})\right) \cong \operatorname{ker}\left(U, p^{*} \nabla+\tau(\nabla)\right)
$$

A method to establish a justification on the nature of the our twisted derived category and their elements as ramifications of a field (to the field equations) is the followed through the Yoneda algebra $[20,21]$ where is searched extends the action of the endomorphism $\operatorname{End}\left(\mathrm{V}_{\text {critical }}\right)$, through the Lie algebra action $\mathrm{g}$, that is the degree zero part that we want, that is to say, the first member of the Penrose transform (6) of their isomorphism must be $H^{0}\left(g[[z]], \mathrm{V}_{\text {critical }}\right)$. We identify in the final part of the demonstration of the theorem 2. 3, that with functions on $\mathrm{Op}_{{ }_{G}}\left(\mathrm{D}^{\times}\right)$, central elements as $I_{F} K$, act via their restriction to the sub-variety $\mathrm{Op}_{L_{G}}$, of opers on $\Sigma$. Then the Yoneda extension algebra must be understood as a projective Harish-Chandra module to the pair $(\mathrm{g}, G[[z]])$, (to $\mathrm{z}$, a singular point of manifold Z). Then $H^{0}\left(g[[z]], \mathrm{V}_{\text {critical }}\right)=\mathrm{C}\left[\mathrm{Op}_{{ }_{G}}\left(\mathrm{D}^{\times}\right)\right]$.

\section{Some Technical Results}

Some technical lemmas can be enounced to establish the fine details on the use of the integral geometrical methods as well as moduli nature kernel of the isomorphism of the Penrose transform in their moduli stacks and the functions in $\mathrm{C}\left[\mathrm{Op}_{{ }_{G}}\left(\mathrm{D}^{\times}\right)\right]$, widely used in the discussions and some arguments of the theory.

Lemma (F. Bulnes) 3. 1. Twisted derived categories corresponding to the algebra of functions $\mathrm{C}\left[\mathrm{Op}_{{ }}\left(\mathrm{D}^{\times}\right)\right]$, are the images obtained by the composition $\mathrm{P}(\mathrm{T})$, on $\tilde{\mathrm{L}}_{\lambda}, \forall \lambda \in \mathrm{h}^{*}$, and such that

$$
\mathrm{P}: H^{0}\left({ }^{L} G, \Gamma\left(\operatorname{Bun}_{\mathrm{G}}, \mathrm{D}^{\times}\right)\right) \cong \operatorname{ker}\left(U, \tilde{\mathrm{D}}_{\lambda, y}\right)
$$

The lemma plays an important role to exhibit the influence of twistor transform to the obtaining the twisted nature of the derived categories $D^{\times}$, starting from the line bundle $L_{\lambda}$.

Proof. It is other form to write the twistor transform treatment followed in [2]. The image that stays is naturally a Penrose transform image.

Using a scheme on functoriality of rings in $\operatorname{Loc}_{{ }{ }_{G}}$, considering the connection scheme to the ramified field in the space-time $M$, we can enounce the isomorphism scheme given by the Yoneda Algebras:

Theorem 6. 1 (F. Bulnes). If we consider the category $M_{\mathrm{K}_{\mathrm{F}}}(\mathrm{g}, \mathrm{Y})$, then a scheme of their spectrum $\mathrm{V}_{\text {critical }}^{\text {Def }}$, where $\mathrm{Y}$, is a Calabi-Yau manifold comes given as:

$$
\operatorname{Hom}_{\hat{\mathrm{g}}}\left(X, \mathrm{~V}_{\text {critical }}^{\text {Def }}\right) \cong \operatorname{Hom}_{\mathrm{Loc}_{L_{G}}}\left(\mathrm{~V}_{\text {critical }}, M_{\mathrm{K}_{F}}(\hat{\mathrm{g}}, \mathrm{Y})\right)
$$

Proof. We use the higher cohomology in the Yoneda algebra context:

$$
H^{\bullet}\left(\mathrm{g}[[z]], \mathrm{g} ; \mathrm{V}_{\text {critical }}\right) \cong \Omega^{\bullet}\left[\mathrm{Op}_{{ }_{G}}(D)\right]
$$

where on left we have the Lie algebra cohomology with coefficients in the vacuum module at critical level [22]:

$$
\mathrm{V}_{\text {critical }}:=U_{\text {critical }} \mathrm{g} \otimes_{\mathrm{g}[[z]]} \mathbf{C}
$$

while on the right side $\mathrm{Op}_{{ }_{G}}(D)$, is a variety of opers on formal disk. The algebra structure suggested byg, is explained by the isomorphism

$$
\begin{gathered}
H^{\bullet}\left(\mathrm{g}[[z]], \mathrm{g} ; \mathrm{V}_{\text {critical }}\right) \cong H^{\bullet}\left(\mathrm{g}[[z]], \mathrm{g} ; \operatorname{End}\left(\mathrm{V}_{\text {critical }}\right)\right) \cong \\
\operatorname{Ext}_{H(c(\hat{G}[[z], G[G] z])}\left(\mathrm{V}_{\text {critical }}, \mathrm{V}_{\text {critical }}\right)
\end{gathered}
$$

where there the right side of (11) is the cohomological group $\operatorname{Hom}_{g}\left(X, \mathrm{~V}_{\text {critical }}^{\text {Def }}\right)$, being that the Ext, groups are computed in a suitable defined category of Harish-Chandra modules as given by $M_{\mathrm{K}_{F}}(\mathrm{~g}, \mathrm{Y})$, that is to say, for the pair $(\mathrm{g}, G[[z]])$, of critical level, and the endomorphism algebra as well as the Lie algebra co-chains must be suitable completed. Then the isomorphism (8) is proved.

\section{Conclusions}

The planting of an isomorphism between two object classes through a scheme theory on functoriality of rings helps to related the geometrical part of the moduli spaces with the corresponding algebraic objects to the Lie algebra structure of the corresponding category of Harish-Chandra modules as given by $M_{\mathrm{K}_{F}}(\mathrm{~g}, \mathrm{Y})$, where the dual Lie algebra is the corresponding to the twisted or "deformable" categories since, the functor Ext", that appear in the demonstration of the Theorem 6. 1, haves $A_{\infty}$-structure which is a deformation of the standard skew-commutative one, deform where said deformation 3 is implemented by degree-scaling. This is a deformation of a commutative algebra based on the cotangent bundle of $\mathrm{Op}_{{ }_{L}}(D)$, and it suffices to show that this deformation is trivial. The only possible deformation is to a non-commutative structure, implemented to the leading order by a Poisson bi-vector field (as could be the deduced by axions defined in other work of this same special issue) along the fibers of $\mathrm{T}^{\vee}\left(\mathrm{Op}_{L_{G}}(D)\right)$.

For other way, the relation between homomorphisms given in (8) can be put in terms of the moduli stack $\operatorname{Bun}_{G}[17]$ (where $\operatorname{Bun}_{G}=G[[z]] / \mathrm{X}, 4$ where $\mathrm{X}$, is the flag variety) as the "quantum" version of the construction of

an algebra symT, (symplectic algebra on cotangent bundles) using the cohomology of the twisted category of $\mathrm{D}^{s}$-modules in (21):

$$
H^{q}\left(\mathrm{Bun}_{G} ; \mathrm{D}^{s}\right)=\mathrm{H}_{G[z z]}^{q}\left(\mathrm{X} ;\left(\wedge^{\bullet} \mathrm{g}\left[\Sigma^{\sigma}\right] \otimes \mathrm{V}_{\text {critical }} ; \partial\right)\right.
$$

where $\partial$, is the Chevalley differential for the fiber-wise Lie algebra action of $\mathrm{g}\left[\Sigma^{\sigma}\right]$, on $\mathrm{V}_{\text {cricical }}$, twisted at the point $\phi \cdot G\left[\Sigma^{\sigma}\right] \in \mathrm{X}$, by the adjoint action of the loop group element $\phi$. Considering that the symbols recovers the "classical" presentation to $H^{q}\left(\mathrm{Bun}_{G} ; \mathrm{Sym}^{r} \mathrm{~T}\right)$,

\section{Nomenclature}

$\begin{array}{ll}\mathrm{T} & \text { Twistor transform. } \\ \mathrm{P} & \text { Penrose transform. }\end{array}$

3 Def. (Deformation). There is a distinguished deformation $\mathrm{D}^{b} \operatorname{Coh}\left(\mathrm{T}^{\vee} A\right)$, [17] the non-commutative deformation of $\mathrm{T}^{\vee} A$, defined by the natural symplectic forms (quantization).

4 Of fact $\operatorname{Bun}_{G}=G[\Sigma] \backslash G((z)) / G[[z]]$. 


\begin{tabular}{|c|c|}
\hline$D^{\times}-$ & $\begin{array}{l}\text { Twisted sheaf of differential operators to } \\
\text { ourOper, given by } \operatorname{Loc}_{{ }^{{ }_{G}}}\left(\mathrm{D}^{\times}\right) \text {. }\end{array}$ \\
\hline$K^{1 / 2}-$ & $\begin{array}{l}\text { Root square of the canonical line bundle on } \\
\text { Bun }_{G} \text {, corresponding to the critical level. } \\
\text { This is a divisor vector bundle. }\end{array}$ \\
\hline $\operatorname{Bun}_{G}(X)-$ & $\begin{array}{l}\text { Category of principal } G-\text { bundles over } \\
C \times X \text {. Also is the moduli stack of principal } \\
G-\text { bundles over } C . \operatorname{Loc}_{t_{G}}\left(\mathrm{D}^{\times}\right)-\text {Set of } \\
\text { equivalence classes of }{ }^{L} G-\text { bundles with a } \\
\text { connection on } \mathrm{D}^{\times} \text {. This space shape a } \\
\text { bijection with the set of gauge equivalence } \\
\text { classes of the ramified operators, as defined } \\
\text { in }[15,16] \text {. }\end{array}$ \\
\hline $\mathrm{D}_{\mathrm{BRST}}-$ & $\begin{array}{l}\text { the derived category on } D \text { - modules of } \\
Q_{\mathrm{BRST}}-\text { operators applied to the geometrical } \\
\text { Langlands correspondence to obtain the } \\
\text { "quantum" geometrical Langlands } \\
\text { correspondence. }\end{array}$ \\
\hline $\mathrm{H}_{G}-\cong(B \backslash G / B)$ & $\begin{array}{l}\text { of bi-equivariant } D-\text { modules on a } \\
\text { complex reductive group } G \text {. }\end{array}$ \\
\hline $\mathrm{D}^{\times}\left(\operatorname{Bun}_{G}(\Sigma)\right)-$ & $\begin{array}{l}\text { It's the category of the twisted Hecke } \\
\text { categories } H_{G, \lambda} \text {. }\end{array}$ \\
\hline$C h_{G,[\lambda]}-$ & $\begin{array}{l}\text { Character sheaves used as Drinfeld centers } \\
\text { in derived algebraic geometry. Their use } \\
\text { connects different cohomologies in the } \\
\text { Hecke categories context. }\end{array}$ \\
\hline $\mathrm{M}_{\text {Higgs }}\left({ }^{L} G, C\right)-$ & $\begin{array}{l}\text { Moduli space of the dualizing of the Higgs } \\
\text { fields, that is to say, quasi-coherent } \\
\text { D-modules. Usuallysaid quasi-coherent }\end{array}$ \\
\hline D- & $\begin{array}{l}\text { modules are coherent D-modules as } \\
\text { D-branes. }\end{array}$ \\
\hline $\mathrm{M}_{\text {Higgs }}(G, C)-$ & $\begin{array}{l}\text { Moduli space of the Higgs fields. Their } \\
\left.\text { fields are the } \theta \in T_{V}^{\vee} B u n_{C}(\Sigma)\right) \text {, }\end{array}$ \\
\hline $\operatorname{End}\left(\mathrm{V}_{\text {critical }}\right)-$ & $\begin{array}{l}\text { Space of endomorphism action through of } \\
\text { extended Verma modules of the Lie algebra } \\
\text { g. }\end{array}$ \\
\hline $\mathrm{Op}_{L_{G}}\left(\mathrm{D}^{\times}\right)-$ & $\begin{array}{l}\text { Space of opers }{ }^{L} G \text {-opers, of the deformed } \\
\text { sheaf } D^{\times} \text {. }\end{array}$ \\
\hline
\end{tabular}

\section{References}

[1] F. Bulnes, Cohomology of Moduli Spaces in Differential Operators Classification to the Field Theory (II), in: Proceedings of Function Spaces, Differential Operators and Non-linear Analysis., 2011, Tabarz Thur, Germany, Vol. 1 (12) pp001-022.

[2] F. Bulnes, Geometrical Langlands Ramifications and Differential Operators Classification by Coherent $D$-Modules in Field Theory, Journal of Mathematics and System Sciences, David Publishing, USA Vol. 3, no.10, pp491-507.

[3] F. Bulnes, Penrose Transform on Induced $D_{G / H^{-}}$Modules and Their Moduli Stacks in the Field Theory, Advances in Pure Mathematics 3 (2) (2013) 246-253. doi: 10.4236/apm.2013.32035.

[4] A. Kapustin, M. Kreuser and K. G. Schlesinger, Homological mirror symmetry: New Developments and Perspectives, Springer. Berlin, Heidelberg, 2009.
[5] F. Bulnes, Integral geometry and complex integral operators cohomology in field theory on space-time, in: Proceedings of 1st International Congress of Applied Mathematics-UPVT (Mexico)., 2009, vol. 1, Government of State of Mexico, pp. 42-51.

[6] F. Bulnes, Penrose Transform on D-Modules, Moduli Spaces and Field Theory, Advances in Pure Mathematics 2 (6) (2012) 379-390. doi: 10.4236/apm.2012.26057.

[7] F. Bulnes, Cohomology of Moduli Spaces on Coherent Sheaves to Conformal Class of the Space-Time, Technical report for XLIII-National Congress of Mathematics of SMM, (RESEARCH) Tuxtla Gutiérrez, Chiapas, Mexico, 2010.

[8] M. Kashiwara and W. Schmid, "Quasi-equivariant $D$-modules, equivariant derived category, and representations of reductive Lie groups, in Lie Theory and Geometry," Progr. Math. vol. 123, Birkhäuser, Boston, 1994, 457-488.

[9] M. Kashiwara, On the maximally over determined systems of linear differential equation I*. Publ. R.I.M.S. Kyoto Univ. 10 (1975) 563-579.

[10] D. Ben-zvi and D. Nadler, The character theory of complex group, 5 (2011) arXiv:0904.1247v2[math.RT],

[11] D. Ben-Zvi, J. Francis, and D. Nadler, Integral transforms and Drinfeld centers in derived algebraic geometry, J. Amer. Math. Soc. 23 (2010) 909-966. doi:arXiv:0805.0157.

[12] E. Frenkel, Lectures on the Langlands Program and conformal field theory, Preprint hep-th/0512172.

[13] A. D'Agnolo and P. Schapira. Radon-Penrose transform for D-modules, J. Funct. Anal. 139 (2) (1996) 349-382.

[14] A. Grothendieck, On the De Rham Cohomology of algebraic varieties, Publ. Math.I.H.E.S. 29 (1966) 95-103.

[15] E. Frenkel, Ramifications of the Geometric Langlands Program, CIME Summer School "Representation Theory and Complex Analysis", Venice, June 2004.

[16] R. Hartshorne, Deformation Theory (Graduate Texts in Mathematics), Springer, USA, 2010.

[17] F. Bulnes (2014) Derived Categories in Langlands Geometrical Ramifications: Approaching by Penrose Transforms. Advances in Pure Mathematics, 4, 253-260. doi: 10.4236/apm.2014.46034.

[18] R. Donagi, T. Pantev, Lectures on the Geometrical Langlands Conjecture and non-Abelian Hodge Theory, 07/01/2008-06/30/2009, , Shing-Tung Yau "Surveys in Differential Geometry 2009", International Press, 2009.

[19] R. Donagi, T. Pantev (2012), Langlands duality for Hitchin systems, Invent.math. arXiv:math/0604617

[20] F. Oort, Yoneda extensions in abelian categories, Mathematische Annalen. 01-1964; 153(3):227-235. DOI: 10.1007/BF01360318

[21] E. Frenkel, C. Teleman, Geometric Langlands correspondence near opers, J. of the Ramanujan Math. Soc. pp. 123-147.

[22] E. Frenkel, C. Teleman, Self extensions of Verma modules and differential forms on opers, Comps. Math. 142 (2006), 477-500. 